\title{
Analysis of Radiation Damage in Magnesium Aluminate Spinel by Means of Cathodoluminescence.
}

Iwona Jozwik $^{1}$, Jacek Jagielski ${ }^{1,2}$, Grzegorz Gawlik ${ }^{1}$, Przemyslaw Jozwik ${ }^{1,2}$, Renata Ratajczak ${ }^{2}$, Gerard Panczer $^{3}$, Nathalie Moncoffre ${ }^{4}$, Anna Wajler ${ }^{1}$ and Agata Sidorowicz ${ }^{1}$.

1. Institute of Electronic Materials Technology, Wolczynska 133, 01-919 Warsaw, Poland.

2. National Centre for Nuclear Research, Soltana 7, 05-400 Otwock/Swierk, Poland.

3. Institut Lumière Matière ILM, UMR5306 Université Lyon 1-CNRS, Université de Lyon, Villeurbanne, France

4. Institut de Physique Nucléaire de Lyon IPNL, Université de Lyon, Université Lyon 1, CNRS/IN2P3, UMR 5822, Villeurbanne, France.

Magnesium aluminate spinel $\left(\mathrm{MgAl}_{2} \mathrm{O}_{4}\right)$ is one of the oxides envisaged to be used in manufacturing of inert matrix fuel. Although the material is recognized for its radiation resistance, most of the experiments were performed on single crystals mainly by the use of Rutherford Backscattering/channeling (RBS/C) method and not much is known about the effects of irradiation of this material in polycrystalline form. The very recent concept is to use luminescence techniques as an experimental method able to measure the level of disorder in polycrystals, as it may be applied to both single and polycrystalline solids.

Damage accumulation was reproduced by using an ion irradiation method, which is the most convenient method of simulation of radiation defects created during the exposure of material to a radiative environment. $\mathrm{MgAl}_{2} \mathrm{O}_{4}$ single crystal samples (commercially available) and polycrystalline samples obtained by the hot-pressing (Astro, Thermal Technology) of magnesium aluminate nanopowder were irradiated with $320 \mathrm{keV} \mathrm{Ar}{ }^{+}$ions at fluencies ranging from $1 \times 10^{12}$ to $2 \times 10^{16} \mathrm{~cm}^{-2}$ in order to create various levels of radiation damage. Cathodoluminescence (CL) measurements were performed using an EMSystems setup mounted in Auriga CrossBeam Workstation (Carl Zeiss NTS). The electron beam energy was reduced to $10 \mathrm{kV}$ in order to limit the analyzed volume of the material as much as possible to the damaged layer $(\sim 200 \mathrm{~nm})$. As the result of measurements carried out, a set of cathodoluminescence spectra has been recorded. The raw signal for each sample with different fluencies of $\mathrm{Ar}^{+}$ions was analyzed in terms of signal intensity of the selected band.

The spectra recorded for polycrystalline samples (Figure 1a) exhibit two broad bands, positioned around $420 \mathrm{~nm}$ and $520 \mathrm{~nm}$ (consisting in fact of a few emission bands). The presence of the first one is assigned to transitions from the excited states to lower energy states of the $\mathrm{Mn}^{2+}$ ion usually present in the synthetic spinels [1]. The second band positioned around $520 \mathrm{~nm}$ is typical for the non-stoichiometric compositions of synthetic spinels, and appears when the vacancy concentration increases [1]. Introduction of the anion vacancies causes a repartitioning of the $\mathrm{Mn}^{2+}$, and the emission at $520 \mathrm{~nm}$ is characteristic of the rearrangement of the activator rather than the direct influence of the vacancies. The intensity of both bands rapidly decrease with the ion irradiation fluence. Similar spectra were observed in the case of monocrystalline samples. Additionally, for single crystals, the damage-build up as a function of accumulated ion fluence was established through RBS/C. The results of CL (reduced signal intensities of the selected bands) and RBS/C analysis for single- and polycrystalline samples were then processed using Multi-Step Damage Accumulation (MSDA) model [2-4], and are presented in Figure 
1b. That allowed for the determination of damage build-up kinetics, and finally cross-section for radiation damage build-up. The phenomena responsible for the rapid decrease of the luminescence of the material are different than those related to the creation of displaced atoms present in the crystal channels. The rate of changes resulting from the formation of non-luminescent recombination centers is very different than that resulting from the creation of single defect clusters or dislocations. Consequently CL technique appears as a complementary tool bringing new possibilities in the damage accumulation studies in single- and polycrystalline materials [5].

\section{References:}

[1] RL Mohler and WB White, Mat. Res. Bull. 29 (10) (1994), p. 1109.

[2] J Jagielski and L Thomé, Vacuum 81 (2007), p. 1352.

[3] J Jagielski and L Thomé, Appl. Phys. A 97 (2009), p. 147.

[4] S. Moll et al, J. Appl. Phys. 106 (2009), p. 073509.

[5] This work was partially sponsored by the National Science Centre (Poland) under the contract number DEC-2011/03/D/ST8/04490 and by 09-133 French - Polish collaboration program.

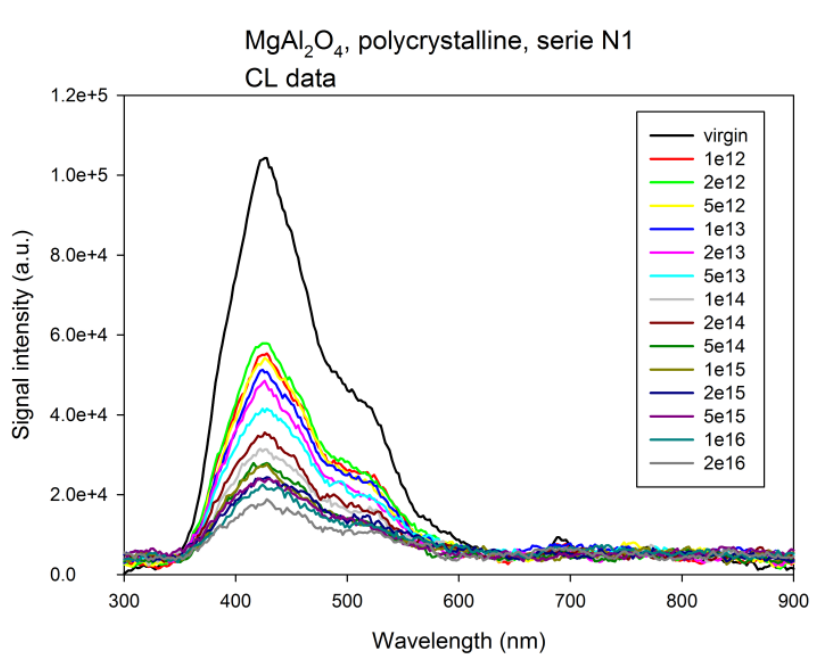

a)

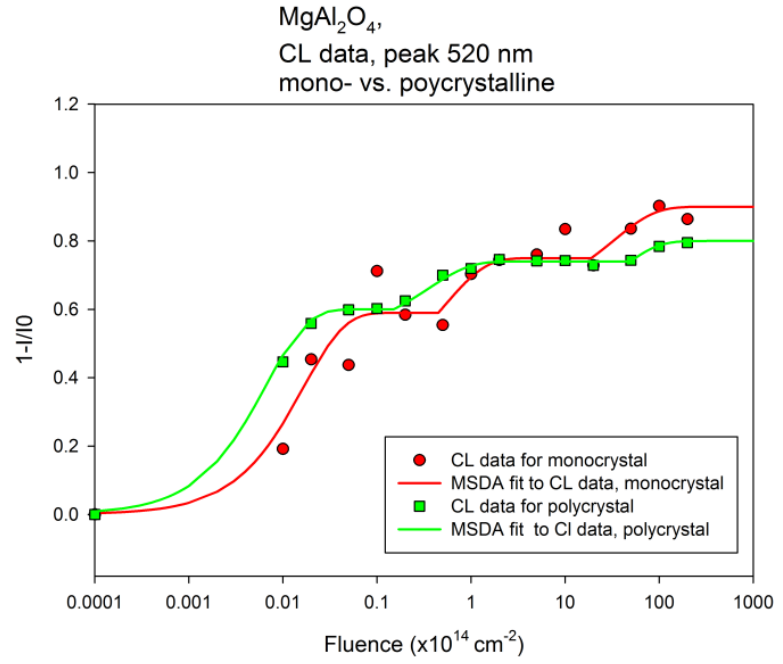

b)

Figure 1. The $\mathrm{CL}$ spectra recorded on $\mathrm{MgAl}_{2} \mathrm{O}_{4}$ polycrystalline samples (a), and radiation damage accumulation kinetics based on CL measurements analyzed with MSDA model for single- and polycrystals of $\mathrm{MgAl}_{2} \mathrm{O}_{4}(\mathrm{~b})$. 